Pacific

Journal of

Mathematics

THE CONSTANT CURVATURE PROPERTY

OF THE WU INVARIANT METRIC

C.K. Cheung and Kang-Tae Kim 


\title{
THE CONSTANT CURVATURE PROPERTY OF THE WU INVARIANT METRIC
}

\author{
C.K. Cheung and Kang-Tae Kim
}

\begin{abstract}
We investigate the property of the $\mathrm{Wu}$ invariant metric on a certain class of psuedoconvex domains. We show that the Wu invariant Hermitian metric, which in general behaves as nicely as the Kobayashi metric under holomorphic mappings, enjoys the complex hyperbolic curvature property in such cases. Namely, the Wu invariant metric is Kähler and has constant negative holomorphic curvature in a neighborhood of the spherical boundary points for a large class of domains in $\mathbb{C}^{n}$.
\end{abstract}

\section{Introduction and the main theorems.}

The invariant metrics including the Carathéodory, Kobayashi, Bergman and Kähler-Einstein metrics (to name only a few) have played an important role in the complex function theory, complex geometry and many related areas of mathematical research. Each invariant metric provides distinct merits and characteristics, and hence its own unique contributions. Among them, the Carathéodory and Kobayashi metrics are exploited mostly because of the distance decreasing property, that all holomorphic mappings are distance decreasing with respect to them. Thus they provide a bridge between the complex function theory and geometry. On the other hand, they are almost never Hermitian, in some sense making them harder to be applied by the differential geometric methods. The Bergman and Kähler-Einstein metrics are Hermitian (in fact Kählerian), and hence they offer wide range of applications in geometric research. However, the distance decreasing property for general holomorphic mappings essentially fail with them. (See $\mathrm{Wu}[7]$ for more detailed discussions on this point.) Along such a circle of reasons, it was natural for $\mathrm{H}$. Wu to introduce the metric that is Hermitian and distance-decreasing for all holomorphic mappings. We call this metric the Wu metric throughout this paper.

This article focuses upon the curvature properties of the Wu metric. As one considers the distance decreasing properties of holomorphic mappings with respect to the given Hermitian/Kählerian metrics, well-known differential geometric generalizations of Schwarz's lemma (see [1], [8] for instance) show that the negativity of curvature can imply the distance decreasing 
property for holomorphic mappings. On the other hand, the Kobayashi metric, while not being Hermitian in general, always enjoys the distance decreasing property. Thus, it was natural for Kobayashi to ask the following question:

Question. Do all Kobayashi hyperbolic manifolds admit a Hermitian metric of negative holomorphic curvature?

Here, by a Kobayashi hyperbolic manifold we mean a complex manifold on which the Kobayashi distance is positive definite. The terminology holomorphic curvature stands for the holomorphic sectional curvature.

Notice that the Hermitian metrics which do not possess distance decreasing property (up to a positive constant multiplier) cannot answer the above question. It appeals to us that hopes, if any, lie with the Wu metric. This has inspired us to consider the curvature behavior of the $\mathrm{Wu}$ metric in various cases.

Indeed, the main focus of this article is centered around the curvature analysis of the $\mathrm{Wu}$ metric on the bounded pseudoconvex domains. In the preceding analysis of the Wu metric on the Thullen domains ([2] and $[\mathbf{3}]$ ), we have observed several totally unexpected aspects of its metric and curvature behavior. When one considers the $\mathrm{Wu}$ metric on the Thullen domain $E_{2}=$ $\left\{\left.(z, w) \in \mathbb{C}^{2}|| z\right|^{2}+|w|^{4}<1\right\}$ for instance, the $\mathrm{Wu}$ metric is real analytic except at points belonging to the subsets

$$
Z=\left\{(z, 0) \in \mathbb{C}^{2}|| z \mid<1\right\} \text { and } M=\left\{\left.(z, w) \in E_{4}|| z\right|^{2}+2|w|^{4}=1\right\} .
$$

Surprisingly, it is $C^{1}$ but not $C^{2}$ at points of $M$. Moreover, the Wu metric is not Kähler "inside" $M$ with a variable holomorphic curvature, whereas it is Kähler "outside" $M$ with a constant holomorphic curvature. It seems that such a "singularity set" for the Wu metric is originated by the weak pseudoconvexity of boundary points of the Thullen domain $E_{4}$. On the other hand, the results of [2] and [3] involve much computation, even for the points outside $M$. As several experts pointed out after [2] and [3] appeared, a simple and straightforward geometric explication of the constant curvature property is desirable. The main point of this paper is exactly focusing upon this; we give a more general and geometric explanation for the boundary constant curvature property of the $\mathrm{Wu}$ metric. As a consequence of our new arguments, we show that the constant curvature property is not only possessed by the complex two dimensional Thullen domains but also shared by much broader a collection of domains that map locally properly onto the ball, for instance. The main results are as follows:

Theorem 1. Let $\Omega$ be a domain obtained by intersecting the ball $B^{n}$ with an open set. Denote the boundaries of $\Omega$ and $B^{n}$ by $\partial \Omega$ and $\partial B^{n}$ respectively. There exists a neighborhood $V$ of $\partial \Omega \cap \partial B^{n}$ such that the Wu metric of $\Omega$ is Kähler with constant negative holomorphic curvautre in $V \cap \Omega$. 
Theorem 2. Let $\widetilde{E}=\left\{\left(z_{1}, \ldots, z_{n}\right) \in \mathbb{C}^{n}:\left|z_{1}\right|^{2 m_{1}}+\cdots+\left|z_{n}\right|^{2 m_{n}}<1\right\}$, where $m_{1}, \ldots, m_{n}$ are integers $\geq 2$. If $p$ is a strongly pseudoconvex boundary point of $\widetilde{E}$, then there exists a neighborhood $V_{p}$ of $p$ such that the Wu metric of $\widetilde{E}$ is Kähler with constant negative holomorphic curvautre in $V_{p} \cap \widetilde{E}$.

Note that Theorem 2 provides a generalization of the complex hyperbolicity results of [2]. Furthermore, its generalization is immediate from the proof; see the remark at the end of Section 5.

Remark. We would like to point out that Theorem 1 cannot be completely localized. The domain $\left\{\left.(z, w) \in \mathbb{C}^{2}|| z\right|^{2}+\sqrt{|w|}<1\right\}$ is locally biholomorphic with the ball in a neighborhood of each smooth boundary point. However, the holomorphic curvature of its $\mathrm{Wu}$ metric is nowhere constant and nowhere Kähler. See [3] for details.

\section{Wu metric and the minimal ellipsoid.}

Let us first recall the definition of the Kobayashi-Royden invariant metric. Let $B^{1}$ be the unit disk in $\mathbb{C}$ and $M$ be a complex manifold. Denote by $H\left(B^{1}, M\right)$ the set of all holomorphic maps from $B^{1}$ to $M$. The KobayashiRoyden metric of $M$ is defined by

$$
k_{M}(p, v)=\inf \{|u|: u \in \mathbb{C} \text { and } d f(0)(u)=v
$$

$$
\text { for some } \left.f \in H\left(B^{1}, M\right) \text { with } f(0)=p\right\}
$$

for all $p \in M$ and $v \in T_{p}(M)$. Here we identify $T_{0}\left(B^{1}\right)$ with $\mathbb{C}$ and || is the euclidean norm.

In [7], Wu introduced a new invariant Hermitian metric. The construction and properties of the $\mathrm{Wu}$ metric are discussed in [2], [5] and [7]. While we point the readers to these articles for a detailed introduction, we include here a brief description of the Wu metric for the Kobayashi hyperbolic manifolds. If $M$ is a Kobayashi hyperbolic complex manifold, then its Wu metric $h_{M}$ and the Kobayashi-Royden infinitesimal metric $k_{M}$ are related as shown in the following proposition.

First let us fix $x \in M$. Let $\alpha$ be a positive definite Hermitian inner product on $T_{x} M$. Denote its unit ball by

$$
B_{\alpha}=\left\{v \in T_{x} M: \alpha(v, v) \leq 1\right\} .
$$

Likewise, consider

$$
I_{x}^{M}=\left\{v \in T_{x} M: k_{M}(x ; v) \leq 1\right\}
$$

which is commonly called the Kobayashi indicatrix of $M$ at $x$. Then, we have: 
Proposition 1 (Wu [7]). The Wu metric $h_{x}$ on $T_{x} M$ satisfies that:

(1) $I_{x}^{M} \subseteq B_{h_{x}}$, and

(2) volume of $B_{h_{x}} \leq$ volume of $B_{g}$ for every $g \in \mathcal{P}_{x}$ that satisfies $I_{x}^{M} \subseteq$ $B_{g}$,

where the volume is measured by any Hermitian inner product on $T_{x} M$ and $\mathcal{P}_{x}$ is the set of all positive definite Hermitian inner products on $T_{x} M$.

In other words, in each tangent space of a Kobayashi hyperbolic manifold, the unit ball of the Wu metric is the unique complex ellipsoid centered at the origin with smallest possible volume among those containing the Kobayashi indicatrix. (See also [4] for the uniqueness property.) In this article, we call this ellipsoid the minimal ellipsoid.

Now we present:

Lemma 1. Suppose $D$ is a subset of $\bar{B}^{n}$. Let $L_{k}=\left\{\left(z_{1}, \ldots, z_{n}\right) \in \bar{B}^{n}\right.$ : $\left|z_{1}\right|^{2} \leq\left|z_{k}\right|^{2}$ and $z_{j}=0$ for $\left.j \neq k\right\}$, where $k=2, \ldots, n$. If $\bigcup_{k=2}^{n} L_{k} \subset D$, then the minimal ellipsoid of $D$ is $\bar{B}^{n}$.

This lemma states that if a domain containing a "sufficiently large portion" of the boundary of the complex unit ball $\partial B^{n}$, then its corresponding minimal ellipsoid is the closed ball $\bar{B}^{n}$.

Proof. Let $E=\left\{\sum_{i} a_{i}\left|z_{i}\right|^{2}+2 \operatorname{Re} \sum_{i \neq j} a_{i j} z_{i} \bar{z}_{j} \leq 1\right\}$, for $a_{i}>0$ with $i, j=$ $1, \ldots, n$, be the minimal ellipsoid that contains $\bigcup_{k=2}^{n} L_{k}$. Assume that $E$ is not the closed ball $\bar{B}^{n}$. Notice that $E$ is not invariant under the action of the group

$$
T=\left\{\left(z_{1}, \ldots, z_{n}\right) \mapsto\left(e^{\sqrt{-1} \theta_{1}}, \ldots, e^{\sqrt{-1} \theta_{n}}\right) \mid \theta_{i} \in \mathbb{R}, i=1, \ldots, n\right\}
$$

unless $a_{i j}=0$. Since the set $\bigcup_{k=2}^{n} L_{k}$ is invariant under the action by $T$ above, and since this action is volume preserving, the uniqueness of the minimal ellipsoid implies that $a_{i j}=0$. The ellipsoid is thus of the form $E=\left\{\sum_{i} a_{i}\left|z_{i}\right|^{2} \leq 1\right\}$. Since $L_{k} \subset E$, we have $a_{1} \geq 1$ and $a_{k} \leq 1$ for $k=2, \ldots, n$. The volume of $E$ is less than that of the $B^{n}$ implies that $a_{1} a_{2} \cdots a_{n}>1$. Hence there exists an integer $j^{\prime}$ between 2 to $n$ such that $a_{1} a_{j^{\prime}}>1$. Without loss of generality, we can assume that $j^{\prime}=2$ with $a_{1} a_{2}>1$. The previous statements can now be reduced to a two dimensional case as follows:

$$
E^{\prime}=\left\{\left(z_{1}, z_{2}\right): a_{1}\left|z_{1}\right|^{2}+a_{2}\left|z_{2}\right|^{2} \leq 1\right\} \supset L_{2}^{\prime}=\left\{\left(z_{1}, z_{2}\right) \in \bar{B}^{2}:\left|z_{1}\right|^{2} \leq\left|z_{2}\right|^{2}\right\} .
$$

This can further imply that in the first quadrant of $\mathbb{R}^{2}$,

$$
E^{\prime \prime}=\left\{a_{1} x+a_{2} y \leq 1\right\} \supset L_{2}^{\prime \prime}=\{x+y \leq 1, x \leq y\} .
$$

But a simple geometrical argument can easily conclude that if $E^{\prime \prime}$ contains $L_{2}^{\prime \prime}$ then $a_{1} a_{2} \leq 1$. This contradicts the assumption that $E$ is not the closed 
unit ball. Since $\bigcup_{k=2}^{n} L_{k} \subset D \subset \bar{B}^{n}$, it follows that the minimal ellipsoid of $D$ is also the closed unit ball.

\section{The extremal discs in $B^{n}$.}

Let $B^{1}$ be the unit disc in $\mathbb{C}$, and $\Omega$ be a domain in $\mathbb{C}^{n}$. A holomorphic map $f: B^{1} \rightarrow \Omega$ is called an extremal mapping at $p \in \Omega$ in the complex direction $v$, if it realizes the Kobayashi-Royden metric at $p$ along $v$. The image, $f\left(B^{1}\right)$, is then called an extremal disc in $\Omega$. See [6].

In this section, we study the behavior of certain extremal discs in $B^{n}$ for points near the boundary.

Consider the point $\widetilde{a}=(a, 0, \ldots, 0) \in B^{n}$. The automorphism $\phi: B^{n} \rightarrow$ $B^{n}$ defined by

$$
\phi\left(w_{1}, \ldots, w_{n}\right) \mapsto\left(\frac{w_{1}+a}{1+\bar{a} w_{1}}, \frac{\sqrt{1-|a|^{2}} w_{2}}{1+\bar{a} w_{1}}, \ldots, \frac{\sqrt{1-|a|^{2}} w_{n}}{1+\bar{a} w_{1}}\right)
$$

sends $(0, \ldots, 0)$ to $\widetilde{a}$. At $(0, \ldots, 0)$, the extremal disc in the direction $\left(X_{1}, \ldots, X_{n}\right) \in \mathbb{C}^{n}$ is given by the complex line $F: B^{1} \rightarrow B^{n}$ with $F(\zeta)=$ $\zeta\left(X_{1}, \ldots, X_{n}\right)$. (Here for simplicity we have assumed that $\left(X_{1}, \ldots, X_{n}\right) \in S$, the unit sphere in $\mathbb{C}^{n}$.) Since the extremal disc is invariant under biholomorphism, the image $\phi \circ F\left(B^{1}\right)$ becomes the extremal disc at $\widetilde{a}$ in the direction of the new vector

$$
(\phi \circ F)^{\prime}(0)=\left(\left(1-|a|^{2}\right) X_{1}, \sqrt{1-|a|^{2}} X_{2}, \ldots, \sqrt{1-|a|^{2}} X_{n}\right),
$$

and the corresponding extremal mapping is

$$
(\phi \circ F)(\zeta)=\left(\frac{\zeta X_{1}+a}{1+\bar{a} \zeta X_{1}}, \frac{\sqrt{1-|a|^{2}} \zeta X_{2}}{1+\bar{a} \zeta X_{1}}, \ldots, \frac{\sqrt{1-|a|^{2}} \zeta X_{n}}{1+\bar{a} \zeta X_{1}}\right) .
$$

If, in addition, $\left|X_{1}\right|^{2} \leq\left|X_{k}\right|^{2}$, for a certain $k>1$ and $X_{j}=0$ for every $j \neq k$, we have $\left|X_{1}\right| \leq \frac{1}{\sqrt{2}}$. The properties of Möbius transformations then implies that the extremal map $(\phi \circ F)(\zeta)$ converges uniformly to $(1,0, \ldots, 0)$ as $a \rightarrow 1$. In other words, we have the following lemma:

Lemma 2. Given a neighborhood $U$ at $(1,0, \ldots, 0)$, there exists a constant $\epsilon>0$ such that if $|1-a|<\epsilon$, then for any $\left(X_{1}, X_{2}, \ldots, X_{n}\right) \in S$ with the property that for certain $k$ between 1 and $n,\left|X_{1}\right| \leq\left|X_{k}\right|$ while $X_{j}=0$ for all $j \neq k$, the extremal disc at $(a, 0, \ldots, 0)$ in the complex direction

$$
\left(\left(1-|a|^{2}\right) X_{1}, \sqrt{1-|a|^{2}} X_{2}, \ldots, \sqrt{1-|a|^{2}} X_{n}\right)
$$

lies inside $U \cap B^{n}$. 


\section{Proof of Theorem 1.}

Suppose $\Omega$ be a domain obtained by intersecting $B^{n}$ with an open set $W$. Let $z \in \Omega=B^{n} \cap W$. Rotating the axis if necessary, we may assume without loss of generality that $z=(a, 0, \ldots, 0)$. By Lemma 2 , if $z$ is close enough to the boundary of $B^{n}$, the extremal discs of $B^{n}$ at $z$ along certain complex directions lie inside $\Omega$. Since $\Omega \subset B^{n}$, the definition of the Kobayashi metric implies that these extremal discs (for $B^{n}$ ) are also extremal for the domain $\Omega$. To state this fact more precisely in terms of Kobayashi indicatrix, we have

$$
\widetilde{L}_{k} \subset I_{z}^{\Omega} \subset I_{z}^{B^{n}}, \text { for } k=2, \ldots, n
$$

where

$$
\widetilde{L}_{k}=\left(\left(1-|a|^{2}\right) Y_{1}, \sqrt{1-|a|^{2}} Y_{2}, \ldots, \sqrt{1-|a|^{2}} Y_{n}\right)
$$

with $\left(Y_{1}, \ldots, Y_{n}\right) \in S$ and $\left|Y_{1}\right| \leq\left|Y_{k}\right|$, while $Y_{j}=0$, for every $j \neq k$. Now, consider the automorphism $\phi: B^{n} \rightarrow B^{n}$ defined in Section 3, that sends $0=(0, \ldots, 0)$ to $z=(a, 0, \ldots, 0)$. By identifying the tangent spaces with $\mathbb{C}^{n}$, we then have

$$
\left(d \phi_{0}\right)^{-1}\left(\widetilde{L}_{k}\right) \subset\left(d \phi_{0}\right)^{-1}\left(I_{z}^{\Omega}\right) \subset\left(d \phi_{0}\right)^{-1}\left(I_{z}^{B^{n}}\right) .
$$

Notice that $\left(d \phi_{0}\right)^{-1}\left(I_{z}^{B^{n}}\right)=\bar{B}^{n}$ and

$$
\left(d \phi_{0}\right)^{-1}\left(\widetilde{L}_{k}\right)=\left\{\left(w_{1}, \ldots, w_{n}\right) \in S:\left|w_{1}\right|^{2} \leq\left|w_{k}\right|^{2} \text { and } w_{j}=0 \text { for } j \neq k\right\} .
$$

Lemma 1 can now be used to conclude that the minimal ellipsoid of $\left(d \phi_{0}\right)^{-1}\left(I_{z}^{\Omega}\right)$ is the same as the closed unit ball. Since the minimal ellipsoids are determined solely by the linear structure, it follows that

$$
\text { minimal ellipsoid of } I_{z}^{\Omega}=I_{z}^{B^{n}} \text {. }
$$

This implies that the $\mathrm{Wu}$ metric of $\Omega$ at this point $z$ coincides with the Poincare-Bergman metric of the ball. Notice that if $p \in \partial \Omega \backslash \partial W \subset \partial B^{n}$, then there exists an open neighborhood $V_{p}$ of $p$ such that the above argument holds for every $z \in V_{p}$. This completes the proof of Theorem 1 .

\section{Proof of Theorem 2.}

Let $m_{1}$ and $m_{2}$ are integers $\geq 2$,

$$
\begin{aligned}
E_{m_{1} m_{2}} & =\left\{\left(z_{1}, z_{2}\right) \in \mathbb{C}^{2}:\left|z_{1}\right|^{2 m_{1}}+\left|z_{2}\right|^{2 m_{2}}<1\right\}, \\
\Sigma & =\left\{\left(z_{1}, 0\right) \in \mathbb{C}^{2}:\left|z_{1}\right| \leq 1\right\} \cup\left\{\left(0, z_{2}\right) \in \mathbb{C}^{2}:\left|z_{2}\right| \leq 1\right\}, \\
\partial \Sigma & =\left\{\left(z_{1}, 0\right):\left|z_{1}\right|=1\right\} \cup\left\{\left(0, z_{2}\right):\left|z_{2}\right|=1\right\} .
\end{aligned}
$$

In this section, we investigate the Wu metric $h_{E}$ of $E=E_{m_{1} m_{2}}$ near $p \in \partial E \backslash \Sigma$. Consider $\Phi\left(z_{1}, z_{2}\right)=\left(z_{1}^{m_{1}}, z_{2}^{m_{2}}\right)$. Then $\Phi$ induces a proper holomorphic mapping from $E_{m_{1} m_{2}}$ onto $B^{2}$ with its branch set $\Sigma$. Take an open neighborhood $U=U_{p}$, say, of $p$ so that $\left.\Phi\right|_{U}$ is 1-1. Let $z \in U \cap E$. By 
Theorem 1, if $z$ is close enough to the boundary point $p$, then the Wu metric of $U^{\prime}=\Phi(U) \cap B^{2}$ at $\Phi(z)$ is the same as the Poincare-Bergman metric of the ball. Denote the Kobayashi indicatrices of $U^{\prime}$ and $B^{2}$ by $I_{\Phi(z)}^{U^{\prime}}$ and $I_{\Phi(z)}^{B^{2}}$, respectively. Then we have

$$
\text { minimal ellipsoid of } I_{\Phi(z)}^{U^{\prime}}=I_{\Phi(z)}^{B^{2}} .
$$

Since the Wu metric is invariant under biholomorphism, it follows that

$$
\text { minimal ellipsoid of } \begin{aligned}
I_{z}^{U \cap E} & =\text { minimal ellipsoid of }\left(d \Phi_{z}\right)^{-1} I_{\Phi(z)}^{U^{\prime}} \\
& =\left(d \Phi_{z}\right)^{-1} I_{\Phi(z)}^{B^{2}} .
\end{aligned}
$$

On the other hand, $\Phi$, being holomorphic, is a distance decreasing mapping with respect to the Kobayashi metrics of $E$ and $B^{2}$. Consequently, we have

$$
I_{z}^{E} \subset\left(d \Phi_{z}\right)^{-1} I_{\Phi(z)}^{B^{2}}
$$

Recall that $U \cap E$ is a subset of $E$. Thus we have

$$
\text { minimal ellipsoid of } I_{z}^{U \cap E} \subset \text { minimal ellipsoid of } I_{z}^{E} \text {. }
$$

Therefore, we can conclude that minimal ellipsoid of $I_{z}^{E}$ equals to $\left(d \Phi_{z}\right)^{-1} I_{\Phi(z)}^{B^{2}}$. In particular, the Wu metric of $E$ at $z$ is given by

$$
h_{E}(z)=\left(\Phi^{*} h_{B^{2}}\right)(\Phi(z)) .
$$

Notice that there exists an open neighborhood $V_{p} \subset U$ of $p$ such that the above argument holds for every $z \in V_{p}$. Now we have

$$
h_{E}=\Phi^{*} h_{B^{2}} \text { at every } z \in V_{p} .
$$

It is evident to see that this argument is also valid for the $n$ dimensional case. Therefore, the proof of Theorem 2 is now complete.

Remark. The same proof can also be used to show the following: Let $\Omega=\left\{\left(z_{1}, z_{2}\right) \in \mathbb{C}^{2}:\left|f_{1}\left(z_{1}, z_{2}\right)\right|^{2}+\left|f_{2}\left(z_{1}, z_{2}\right)\right|^{2}<1\right\}$, where $\left(f_{1}, f_{2}\right): \Omega \rightarrow$ $B^{2}$ is a proper holomorphic mapping. Then, the set of boundary points away from branch locus of the proper holomorphic mapping admits an open neighborhood on which the Wu metric is complex hyperbolic.

Acknowledgments. It is our pleasure to acknowledge our indebtedness to $\mathrm{H}$. Wu for his encouragements, interest and many helpful discussions with the authors. 


\title{
References
}

[1] L. Ahlfors, An extension of Schwarz's Lemma, Trans. Amer. Math. Soc., 43 (1938), 359-364, CMP 1501 949, Zbl 0018.41002.

[2] C.K. Cheung and K.T. Kim, Analysis of the Wu metric I: The case of convex Thullen domain, Trans. Amer. Math. Soc., 358 (1996), 1429-1457, MR 96i:32026, Zbl 0872.32017.

[3] Analysis of the Wu metric II: The case of non-convex Thullen domain, Proc. Amer. Math. Soc., 125 (1997), 1131-1142, MR 97f:32029, Zbl 0872.32018.

[4] F. John, Extremum problems with inequalities as subsidiary conditions, Studies and Essays Presented to Richard Courant on his 60th Birthday, January 8, 1948, Interscience, New York, 1948, 187-204, MR 10,719b, Zbl 0034.10503.

[5] K. Kim, The Wu metric and minimal ellipsoids, Proceedings of the 3rd Pacific Rim Geometry Conference, (J. Choe ed.), Monogr. Geom. Topology, 25, International Press, Cambridge, 1998, 121-138, MR 2001f:32017.

[6] L. Lempert, La métrique de Kobayashi et la representation des domains sur la boule, Bull. Soc. Math. France, 109 (1981), 427-474, MR 84d:32036, Zbl 0492.32025.

[7] H. Wu, Old and new invariant metrics, Several complex variables: Proc. of MittagLeffler Inst., 1987-88 (J.E. Fornaess ed.), Math. Notes, Princeton Univ. Press, 38 (1993), 640-682, MR 94a:32038, Zbl 0773.32017.

[8] S.-T. Yau, A general Schwarz lemma for Kähler manifolds, American J. Math., 100 (1978), 197-203, MR 58 \#6370, Zbl 0424.53040.

Received March 9, 2000. Research of the second named author is supported in part by POSTECH Special Fund and KOSEF Grant 981-0104-018-2 and 1999-2-102-003-5 of The Republic of Korea.

\author{
Department of Mathematics \\ Boston College \\ Chestnut Hill, MA 02467 \\ E-mail address: ck.cheung@bc.edu \\ Department of Mathematics \\ Pohang University of Science and Technology \\ POHANG 790-784 \\ The Republic of Korea \\ E-mail address: kimkt@postech.edu
}

